\title{
Das sind die Ad-hoc-Änderungen beim Labor
}

_ Nach Erhebungen der KBV steigen die Ausgaben für Laborleistungen um jährlich etwa $4,7 \%$ an. Bei einer durchschnittlichen jährlichen Steigerung der Gesamtvergütung um $2,3 \%$ wird deshalb sukzessive Geld in diesen Bereich umgelenkt, das eigentlich für die unmittelbare Versorgung der Patienten vorgesehen ist. Diese Dynamik entsteht laut KBV, weil die Zahl der Patienten, die Laborleistungen erhalten, um etwa $2,6 \%$ und die Zahl der Behandlungsfälle um 3,5\% steigen. Angeblich wird diese Dynamik durch alle Arztgruppen verursacht.

\section{MMW-KOMMENTAR}

Da die AG "Weiterentwicklung Labor" (siehe Meldung links) keine einfachen Maßnahmen zur Mengenbegrenzung finden konnte und auch keinen Spielraum für eine Absenkung der Bewertung von Laborleistungen sieht, soll es nun in gestufter Form "Hilfsmaßnah- men" geben. Kurzfristig ist beabsichtigt, das von Fachärzten erbrachte Labor und die fachärztlichen Laborgrundpauschalen exklusiv dem fachärztlichen Vergütungsanteil zuzuordnen. Das sogenannte Praxislabor soll veranlasserbezogen dem hausärztlichen oder fachärztlichen Vergütungsanteil zugeschlagen werden.

Damit würden aus dem „Grundbetrag Labor" nur noch Leistungen von Laborärzten, von Laborgemeinschaften und der Wirtschaftlichkeitsbonus (Nr. 32001 EBM) vergütet. Steigt diese Menge allerdings weiterhin, müsste auch weiter gesetzeswidrig Geld auch aus dem hausärztlichen Vergütungsanteil zugeschossen werden. Deshalb soll nun der Wirtschaftlichkeitsbonus angehoben werden, um einen Anreiz zu schaffen, beim Labor sparsamer zu sein. Auch könnten die Kennziffern abgeschafft werden, mit denen bei bestimmten Erkrankungen die Laborleistungen nicht mehr für den Wirtschaftlich- keitsbonus zählen. Für die Bonus-Berechnung könnte auch die Differenzierung nach Leistungen des Allgemeinlabors (EBM-Kapitel 32.2) und des Speziallabors (Kapitel 32.3) fallen.

Mittelfristig könnten "diagnostische Pfade" fürs Labor vorgegeben und das Spektrum veranlasster Laborleistungen arztgruppenbezogen begrenzt werden. Hausärzte könnten dann bei bestimmten Erkrankungen nur noch vorgegebene Laborleistungen im Rahmen eines Gesamtspektrums anfordern. Bei Abweichungen müssten die Patienten an Fachärzte überwiesen werden.

Wohl wegen des bürokratischen Aufwands denkt man in der AG darüber nach, Laborleistungen langfristig in die individuellen Praxiskosten einzubeziehen. Dies würde bedeuten, dass die Praxen Laborleistungen künftig wie andere Verbrauchsartikel beziehen, selbst bezahlen und damit dem eigenen unternehmerischen Risiko unterwerfen.

\section{Selbst wenn Patienten drängeln, können sie später Schadensersatz verlangen}

Ein Arzt muss eine Behandlung, die gegen medizinische Standards verstößt, ablehnen. Dies gilt auch dann, wenn der Patient die Behandlung ausdrücklich verlangt. Selbst eine umfassende ärztliche Aufklärung schützt in diesen Fällen nicht vor Schadensersatzforderungen, sollte die Behandlung zu Problemen führen. Das hat das Oberlandesgericht Hamm entschieden (Az.: 26 U 116/14).

\section{MMW-KOMMENTAR}

Der zugrunde liegende Fall stammt aus dem zahnärztlichen Bereich, kann aber auf alle anderen medizinischen Sektoren übertragen werden. Ein Zahnarzt stellte bei einer Patien- tin eine Funktionsstörung der Kiefergelenke fest. Er empfahl, diese mit einer Schiene zu therapieren und danach die Seitenzähne zu stabilisieren. Erst im Anschluss wollte er mit der Sanierung der Frontzähne beginnen. Weil die Patientin aber darauf bestand, begann er schon vor Abschluss der Therapie mit der Frontzahnsanierung. Das Resultat war eine Kompression der Kiefergelenke der Patientin. Diese verlangte daraufhin wegen einer fehlerhaften Behandlung u.a. 25.000 Euro Schmerzensgeld sowie die Rückzahlung des geleisteten Honorars. Das zuständige Landgericht stellte die Ersatzpflicht des Beklagten fest. Die Berufung vor dem Oberlandesgericht blieb ohne Erfolg.

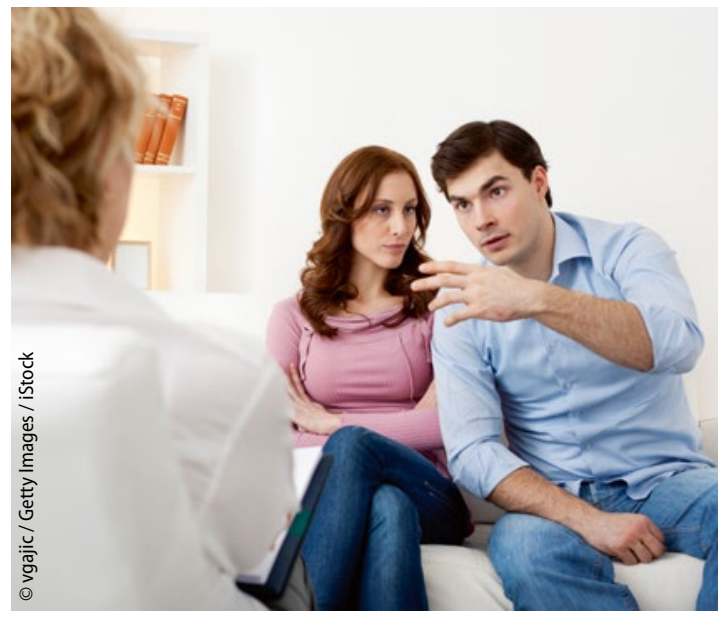

Nicht zu einer Therapie drängen lassen! 